\title{
Announcement of New Section Editors
}

KJR recently established the thyroid section as an independent section of KJR. We are pleased to announce that Dr. Dong Gyu Na begins to serve as a new editor of thyroid section. We also announce that Dr. Sang Hyun Suh of Yonsei University has been appointed as a new section editor for the neurointervention section, replacing Dr. Woong Yoon. We appreciate Dr. Yoon's dedicated service as a section editor for a long period (from April 2012 to December 2018).

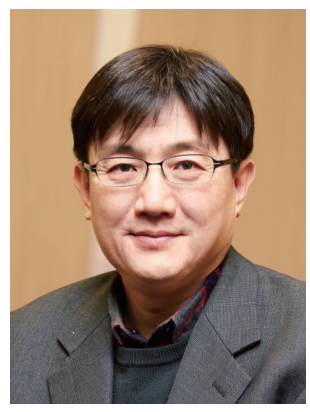

New Thyroid Section Editor, Dr. Dong Gyu Na

Dr. Dong Gyu Na graduated from Seoul National University, School of Medicine in 1987. He completed internship and residency training in the department of radiology at Seoul National University Hospital. He worked as an associate professor in the neuroradiology section at Samsung Medical Center until 2003 and then worked as a clinical professor in the neuroradiology and thyroid radiology section at Seoul National Hospital until 2008. He worked as the director of neuroradiology and thyroid radiology section in Human Medical Imaging and Intervention Center since 2008, and he became a clinical professor and the director of thyroid radiology section at Gangneung Asan Hospital in 2017. Dr. Dong Gyu Na served as the president of Korean Society of Thyroid Radiology (KSThR) from 2010 until 2014 and he currently is the chair of the Clinical Guideline Committee of KSThR. He has more than 120 SCI(E) scientific publications to his credit, as well as 6 book chapters. He has operated outpatient thyroid clinic since 2008 and has tried to develop the role of radiologists in the management of patients with thyroid nodules. His major clinical and research field is the imaging diagnosis and interventional treatment of thyroid cancer.

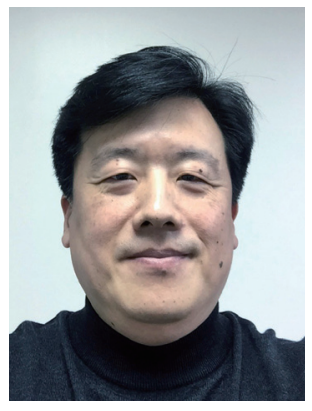

\section{New Neurointervention Section Editor, Dr. Sang Hyun Suh}

Dr. Sang Hyun Suh graduated from Yonsei University, college of medicine, where he had MD degree in 1995. He received residency training in radiology in the Severance Hospital, Yonsei University and he was certified in the Diagnostic Radiology by the Korean Board of Radiology in 2003. Subsequently, he received fellowship training in the interventional radiology and neuroradiology for three years at the same hospital and became a faculty member of the Department of Radiology at Yongdong Severance Hospital, Yonsei University, as a clinical assistant professor in 2006. Since March 2016, he has been serving as a professor of the Department of Radiology at the Gangnam Severance Hospital, Yonsei University.

He has been a reviewer for several journals, including the Korean Journal of Radiology, American Journal of Neuroradiology, and Cardiovascular Interventional radiology. He authored more than 116 scientific publications including $85 \mathrm{SCI}(\mathrm{E})$ publications as well as two book chapters for neuroradiology and neurointervention. He is actively participating both regional and international meetings giving many scientific presentations in neurointervention since 2005. 